University of Nebraska - Lincoln

DigitalCommons@University of Nebraska - Lincoln

Faculty Publications from the Department of Electrical \& Computer Engineering, Department Electrical and Computer Engineering

2012

\title{
Imbalance Fault Detection of Direct-Drive Wind Turbines Using Generator Current Signals
}

Xiang Gong

University of Nebraska-Lincoln, xiang.gong@huskers.unl.edu

Wei Qiao

University of Nebraska-Lincoln, wqiao@engr.unl.edu

Follow this and additional works at: https://digitalcommons.unl.edu/electricalengineeringfacpub

Part of the Electrical and Computer Engineering Commons

Gong, Xiang and Qiao, Wei, "Imbalance Fault Detection of Direct-Drive Wind Turbines Using Generator Current Signals" (2012). Faculty Publications from the Department of Electrical and Computer Engineering. 167.

https://digitalcommons.unl.edu/electricalengineeringfacpub/167

This Article is brought to you for free and open access by the Electrical \& Computer Engineering, Department of at DigitalCommons@University of Nebraska - Lincoln. It has been accepted for inclusion in Faculty Publications from the Department of Electrical and Computer Engineering by an authorized administrator of DigitalCommons@University of Nebraska - Lincoln. 


\title{
Imbalance Fault Detection of Direct-Drive Wind Turbines Using Generator Current Signals
}

\author{
Xiang Gong, Student Member, IEEE, and Wei Qiao, Member, IEEE
}

\begin{abstract}
Imbalance faults constitute a significant portion of all faults in wind turbine generators (WTGs). WTG imbalance fault detection using generator current measurements has advantages over traditional vibration-based methods in terms of cost, implementation, and system reliability. However, there are challenges in using current signals for imbalance fault detection due to low signal-to-noise ratio of the useful information in current signals and nonstationary characteristic frequencies of imbalance faults. This paper proposes a method of using generator stator currents for imbalance fault detection of direct-drive WTGs. In the proposed method, the variable shaft rotating frequency of a WTG is estimated from one phase stator current measured from the generator terminal by using a phase-locked loop method. The estimated shaft rotating frequency is then processed by using appropriate upsampling and variable-rate downsampling algorithms. Consequently, the variable characteristic frequencies of imbalance faults in the spectrum of the estimated shaft rotating frequency are converted to constant values. Therefore, the signatures of wind turbine imbalance faults can be clearly identified from power spectral density analysis of the converted shaft rotating frequency signal. Simulation and experimental results show that the proposed method is effective to detect various imbalance faults in direct-drive WTGs.
\end{abstract}

Index Terms-Current signal, direct-drive wind turbine, fault detection, imbalance fault, power spectral density (PSD).

\section{INTRODUCTION}

$\mathbf{T}$ HE PENETRATION of wind power has increased greatly over the last decade in the U.S. and across the world. America's wind power industry installed $1118 \mathrm{MW}$ of new capacity in the first quarter of 2011 alone and entered the second quarter with another $5600 \mathrm{MW}$ under construction [1]. As the number of wind turbines continues to grow, it becomes more and more challenging for engineers to do inspection and maintenance for the wind turbines.

Imbalance faults constitute a significant portion of all faults in wind turbine generators (WTGs) [2]. A common imbalance fault in WTGs is shaft/blade imbalance. A blade imbalance can be caused by errors in manufacturing and construction, icing, deformation due to aging, or wear and fatigue during the operation of the WTG. Components tend to shift and wear in

Manuscript received September 28, 2011; revised January 13, 2012; accepted February 15, 2012. Date of publication March 22, 2012; date of current version May 18, 2012. This work was supported in part by the U.S. Department of Energy under Grant DE-EE0001366 and the Nebraska Center for Energy Sciences Research. Paper no. TEC-00504-2011.

The authors are with the Department of Electrical Engineering, University of Nebraska-Lincoln, Lincoln, NE 68588-0511 USA (e-mail: xiang.gong@ huskers.unl.edu; wqiao@engr.unl.edu).

Color versions of one or more of the figures in this paper are available online at http://ieeexplore.iee.org.

Digital Object Identifier 10.1109/TEC.2012.2189008 varying degrees over time, causing imbalance on the rotating shaft/blades. Another common imbalance fault is aerodynamic asymmetry, which can be caused by several factors, including high wind shear and errors in the control mechanism. If the pitch of one blade is slightly different from the other two blades due to errors in the control mechanism, the torque on the rotating shaft will not be balanced, leading to aerodynamic asymmetry. A small imbalance fault can cause significant consequences on the towers and the WTGs. For instance, a blade imbalance caused by icing can create additional loads on the supporting tower of the wind turbine, which may lead to fractures and possible collapses [3] of the tower. Due to WTGs' delicate structure and high repairing cost [2], [4], [5], effective imbalance fault detection is of significant interest to the wind power industry.

Most existing methods for imbalance fault detection require additional mechanical sensors and data acquisition equipment to implement [6]-[9]. The most commonly used sensors are vibration sensors, such as accelerometers. These sensors are mounted on the surface of WTG components, which are situated on high towers and are difficult to access during WTG operation. Moreover, the sensors and equipment are inevitably subject to failure, which could cause additional problems with system reliability and additional operating and maintenance costs. Current-based (mechanical-sensorless) fault detection techniques use generator current measurements that have been used by the control system of the WTG; no additional sensors or data acquisition equipment is needed. Moreover, current signals are reliable and easily accessible from the ground without intruding the WTGs. Therefore, current-based fault detection techniques have great economic benefits and potential to be adopted by the wind power industry.

However, there are challenges in using current signals for imbalance fault detection. First, the characteristic frequencies of imbalance faults depend on the shaft rotating frequency, which is known as the 1P frequency [10], [11]. As mentioned in [6], since the 1P frequency of a WTG usually varies with wind speed, it is a problem to extract the imbalance fault signatures from the nonstationary stator current signals of the WTG using traditional spectrum analysis methods. Moreover, the dominant component of a current signal is its fundamental-frequency component. Therefore, the useful information in current signals for imbalance fault detection usually has a low signal-to-noise ratio, which makes the fault detection difficult.

Several signal processing techniques, e.g., the classical power spectral density (PSD) analysis [7], [8], bicoherence analysis [10], time-frequency analysis [9], [12], amplitude demodulation [13], [14], and data mining [15], have been used for imbalance fault detection of WTGs. The classical PSD analysis 
and bicoherence analysis can identify imbalance faults based on their characteristic frequencies. However, this capability is only available for stationary signals. The time-frequency analysis and amplitude demodulation methods are able to extract fault signatures from nonstationary signals. However, they cannot clearly identify imbalance faults from interferences that have similar patterns as the faults in the time or frequency domain. Furthermore, these methods usually have low resolution and require high computational resources compared to the PSD analysis [13]. Data mining has been applied for wind turbine condition prediction using the records of maintenance. However, no work has been reported on WTG imbalance fault detection via data mining on current signals. Therefore, a computationally efficient, highly sensitive signal processing method is desired for current-based online imbalance fault detection for WTGs operating in nonstationary conditions.

This paper proposes a novel method of using generator stator current signals for online imbalance fault detection of directdrive wind turbines. The proposed method first estimates the variable shaft rotating frequency of a WTG from the measured one phase stator current of the generator, and then processes the estimated shaft rotating frequency signal in a way such that the variable characteristic frequencies of imbalance faults become constant values in the PSD of the processed shaft rotating frequency signal. The proposed method is validated by simulation and experimental studies for detection of shaft/blade imbalance and aerodynamic asymmetry of direct-drive wind turbines under variable-speed operating conditions.

\section{Characteristic FreQuencies of Imbalance Faults}

It has been reported that the spectra of the shaft torque and the output electric power of a WTG with three blades are determined by certain events. The vibration at $3 \mathrm{P}$ frequency, which is three times the shaft rotating frequency of a WTG, is generated by the effect of yaw error, wind shear, or tower shadow [11], [16]. The vibration at $1 \mathrm{P}$ frequency is created by imbalance faults, including blade imbalance and aerodynamic asymmetry [6], [10], [17]. Moreover, these events also affect the shaft rotating frequency of the WTG and produce excitations at the corresponding frequencies in the shaft rotating frequency signals.

The shaft system of a direct-drive WTG can be represented by a one-mass model. The motion equation is given by

$$
\begin{aligned}
J \cdot\left[\frac{d \omega_{r}(t)}{d t}\right] & =T_{t}(t)-T_{e}(t)-D \cdot \omega_{r}(t) \\
\omega_{r}(t) & =2 \pi \cdot f_{r}(t)
\end{aligned}
$$

where $J$ is the total inertia constant of the WTG; $\omega_{r}$ is the angular shaft rotating speed of the WTG; $f_{r}$ is the WTG shaft rotating frequency, which is equal to the $1 \mathrm{P}$ frequency; $d \omega_{r}(t) / d t$ is the angular acceleration; $T_{t}$ and $T_{e}$ are the torques of the turbine and the generator, respectively; and $D$ is the damping coefficient. Since this is a linear model which presents the relationship between the shaft rotating speed and the torques of a WTG, if there is a vibration in the torques of the WTG, an excitation with the same frequency will appear in the shaft rotating speed signal.

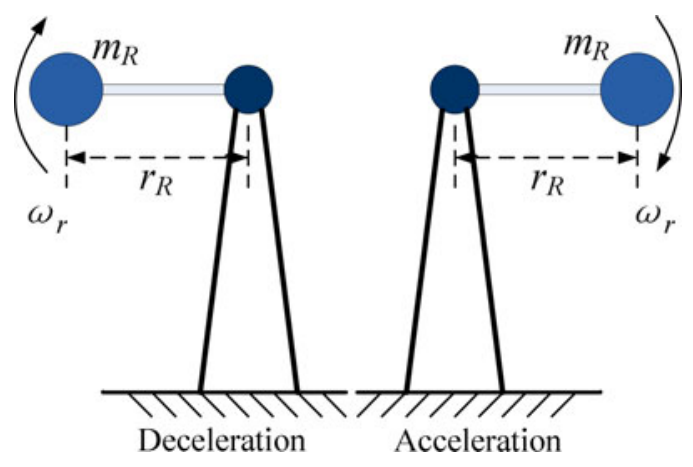

Fig. 1. Effect of blade imbalance of a wind turbine.

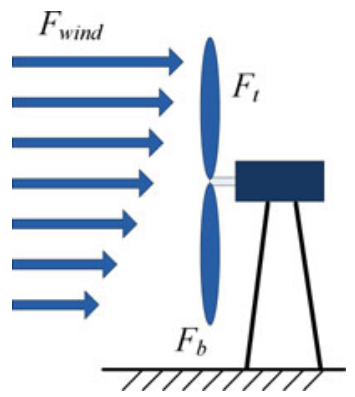

Fig. 2. Effect of aerodynamic asymmetry of a wind turbine caused by wind shear.

When an imbalance fault occurs on the shaft of a WTG, an additional force will be induced in the shaft. In the case of blade imbalance where the mass distribution of one blade is different from others, a rotor mass imbalance will occur and induce vibrations in the shaft rotating speed of the WTG. This is illustrated in Fig. 1, where $m_{R}$ is the equivalent imbalance mass, and $r_{R}$ is the distance between the equivalent imbalance mass and the center of the shaft. When the equivalent imbalance mass rotates from the top to the bottom of the rotating plane, the power of gravity accelerates the shaft. On the other hand, when the equivalent imbalance mass rotates from the bottom to the top of the rotating plane, the power of gravity decelerates the shaft. Consequently, the shaft rotating speed vibrates at the frequency of $1 \mathrm{P}$.

Aerodynamic asymmetry stands for that the force affected on one blade is different from those on other blades. Aerodynamic asymmetry along with yaw error, wind shear, or tower shadow together influences the shaft rotating speed of a WTG. For example, Fig. 2 shows the effect of an aerodynamic asymmetry caused by wind shear, where $F_{\text {wind }}$ is the force of the wind flow affected on the blades, $F_{t}$ is the force of the wind flow affected on the blade that is on the top of the rotating plane, and $F_{b}$ is the force of the wind flow affected on the blade that is on the bottom of the rotating plane. The amplitude of $F_{t}$ is always greater than that of $F_{b}$ due to the effect of wind shear, which follows the following power law:

$$
\frac{U(z)}{U\left(z_{r}\right)}=\left(\frac{z}{z_{r}}\right)^{\alpha}
$$




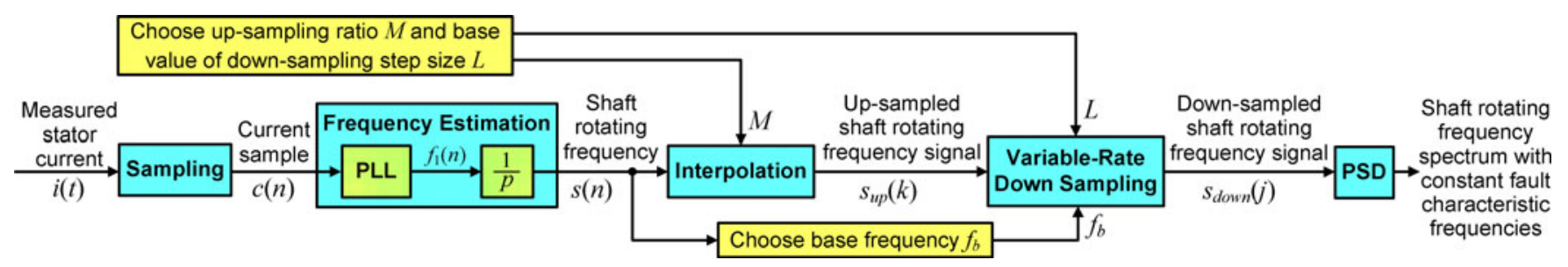

Fig. 3. Schematic diagram of the propose method.

where $U(z)$ and $U\left(z_{r}\right)$ are wind velocities at height $z$ and the reference height $z_{r}$, respectively, and $\alpha$ is the power law exponent [18].

Normally, a blade has the largest acceleration caused by $F_{t}$ and the smallest acceleration caused by $F_{b}$. Therefore, a vibration at the $3 \mathrm{P}$ frequency is produced in the shaft speed by wind shear in a balanced wind turbine with three blades. In the case of an aerodynamic asymmetry, a blade of the turbine has different $F_{t}$ and $F_{b}$ from the other two blades. As a result, the acceleration and deceleration of the imbalanced blade produce a vibration at the $1 \mathrm{P}$ frequency in the shaft speed. On the other hand, the other two blades have different $F_{t}$ and $F_{b}$ from the imbalanced one. As a result, a vibration also appears at the $2 \mathrm{P}$ frequency in the shaft speed signal.

The characteristic frequencies of shaft/blade imbalance and aerodynamic asymmetry both appear at the $1 \mathrm{P}$ frequency in the shaft speed signal of a wind turbine. Therefore, the excitations of the shaft speed signal at the $1 \mathrm{P}$ frequency can be used as a signature for imbalance fault detection.

\section{Proposed Method For Fault Signature Extraction}

In the proposed method, only one phase stator current is measured for imbalance fault detection; the shaft rotating frequency is estimated from the measured current signal. In a direct-drive wind turbine, the relationship between the shaft rotating frequency $f_{r}$ and the fundamental frequency $f_{1}$ of the stator current is given as follows:

$$
f_{1}(t)=p \times f_{r}(t)
$$

where $p$ is the number of pole pairs of the generator. A phaselocked loop (PLL) model is designed in the proposed method to estimate $f_{1}$ from the stator current measurement of the WTG. Then, the shaft rotating frequency $f_{r}$ is obtained from (4).

Since the imbalance fault characteristic frequency of $1 \mathrm{P}$ usually varies with wind speed during maximum power point tracking [19], it is difficult to extract the fault signature from the nonstationary stator current signals of a WTG using classical spectrum analysis methods [6]. However, as mentioned in [6], if a wind turbine rotates at a constant speed, the classical PSD analysis could identify an imbalance fault effectively based on its characteristic frequency. Therefore, if the estimated shaft rotating frequency signal is preprocessed in such a way that the variable $1 \mathrm{P}$ frequency (i.e., a characteristic frequency of imbalance faults) of the WTG is converted to a fixed value, the classical PSD analysis can then be used to detect the imbalance faults for a variable-speed wind turbine.
Define $\Omega_{r}$ the normalized frequency of the shaft rotating frequency or 1P frequency signal, which is estimated from the stator current signal, and define $f_{s}$ the sampling frequency of the stator current. The relationship among $f_{r}, f_{s}$, and $\Omega_{r}$ can be written as

$$
\frac{\Omega_{r}(t)}{2 \pi}=\frac{f_{r}(t)}{f_{s}}
$$

where $\Omega_{r}(t)$ is expected to be constant in the PSD analysis. Therefore, if the sampling frequency $f_{s}$ is changed continuously with $f_{r}(t)$ to make the right-hand side of (5) constant, $\Omega_{r}(t)$ will become constant. The proposed method preprocesses the estimated shaft rotating frequency signal of a direct-drive WTG to obtain a constant $\Omega_{r}(t)$, which is shown in Fig. 3 and implemented in the following steps.

1) Choose an upsampling ratio $M$ and a base value of the downsampling step size $L$.

2) Sample the measured nonstationary current $i(t)$ of the WTG with a fixed sampling rate; the result is $c(n)$, where $n=1,2,3, \ldots, N$, and $N$ is the length of the stator current record.

3) Estimate the variable fundamental frequency $f_{1}(n)$ of the nonstationary stator current signal $c(n)$ by using a PLL method.

4) Divide the estimated $f_{1}(n)$ by the pole pair number $p$ to obtain the shaft rotating frequency signal $s(n)$ and choose a base frequency $f_{b}$ based on $s(n)$.

5) Upsample (interpolate) $s(n)$ by an constant upsampling ratio of $M$; the result is $s_{\text {up }}(k)$, where $k=1,2,3, \ldots, M \times$ $N$.

6) Downsample $s_{\text {up }}(k)$ by a variable downsampling step size; the result is $s_{\text {down }}(j)$, where $j=1,2,3, \ldots, J$, and $J$ is determined by $M, N$, and $L$. In the downsampling process

$$
s_{\text {down }}(1)=s_{\text {up }}(1) .
$$

If $s_{\text {down }}(j)=s_{\text {up }}(k)$, then

$$
s_{\text {down }}(j+1)=s_{\text {up }}\left(k+\text { round }\left[\frac{L \cdot f_{b}}{s_{\text {up }}(k)}\right]\right)
$$

where round $\left[L \cdot f_{b} / s_{\text {up }}(k)\right]$ is the variable downsampling step size, which depends on the upsampled shaft rotating frequency $s_{\text {up }}(k)$; and round(·)stands for rounding a number to the nearest integer. The downsampling process to obtain $s_{\text {down }}(j)$ is equivalent to resampling the original or upsampled shaft rotating frequency signal $\left[s(n)\right.$ or $\left.s_{\text {up }}(k)\right]$ with a variable sampling frequency $f_{s}(j)$, whose value is proportional to the value of $s_{\text {down }}(j)$. According to (5), the 


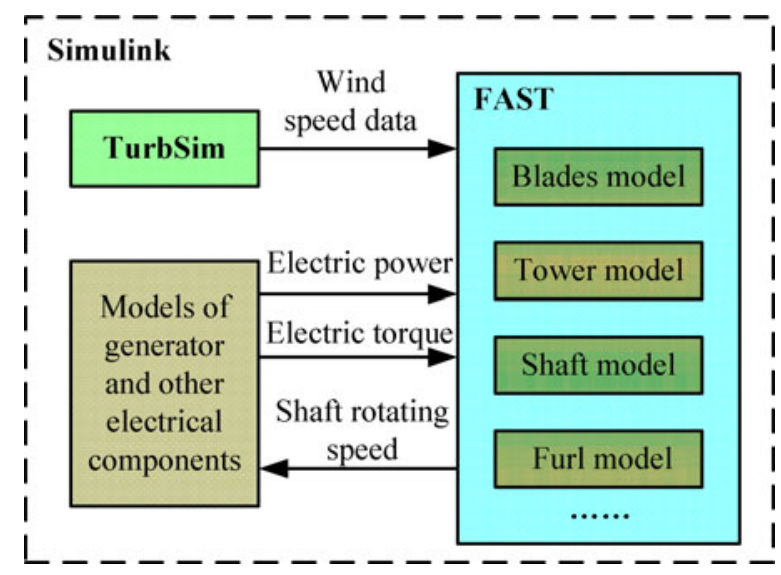

Fig. 4. Structure of the WTG model with wind data.

normalized frequency of $s_{\text {down }}(j)$, which is $\Omega_{\text {down }}(j)$, is given by

$$
\frac{\Omega_{\text {down }}(j)}{2 \pi}=\frac{s_{\text {down }}(j)}{f_{s}(j)}
$$

where $\Omega_{\text {down }}(j)$ is now a constant value.

7) Calculate the classical PSD of the downsampled estimated shaft rotating frequency $s_{\text {down }}(j)$ to extract the fault signature, which now has a constant characteristic frequency.

By using the proposed method, the variable characteristic frequency of $1 \mathrm{P}$ of imbalance faults becomes a constant value in the PSD of $s_{\text {down }}(j)$. Therefore, the resulting PSD is called the 1P-invariant PSD, and the magnitude of the excitation at the 1P frequency in the PSD of $s_{\text {down }}(j)$ can be used as a signature to clearly identify imbalance faults. In the proposed method, the constant base value of the downsampling step size $L$ should be chosen based on two criteria. First, $L$ should be large enough to eliminate the quantization error due to the requirement of an integral downsampling step size. Second, $L$ should be small enough to ensure that the sampling frequency after downsampling is greater than twice the $1 \mathrm{P}$ frequency. Normally, $L$ should be larger than 10 . The base frequency $f_{b}$ is chosen to be the mean value of the estimated shaft rotating rotating frequency $s(n)$. Furthermore, if the measured current is sampled with a sufficiently high sampling rate in Step 2 such that the sampling frequency of the downsampled signal $s_{\text {down }}(j)$ without using upsampling is greater than twice the characteristic frequency of the imbalance faults, then Step 5 is not necessary.

\section{Simulation Study of Imbalance Fault Detection}

\section{A. WTG Model}

A dynamical model of a 10-kW direct-drive WTG system is built in a FAST (Fatigue, Aerodynamics, Structures, and Turbulence) [20] and Simulink combined environment, as illustrated in Fig. 4. FAST version 7.0 is used to simulate the dynamics of the wind turbine, while Simulink is used to simulate the generator and other electrical components of the WTG system.

FAST is an aeroelastic code developed initially by Oregon State University. It is one of the most advanced design codes for horizontal-axis wind turbines. In the simulation study, FAST works as a subroutine in Simulink. The signals of the electric power, electric torque, and shaft rotating speed are used to connect the FAST and Simulink models of the WTG system.

The model wind turbine in FAST mainly includes tower, blades, shaft, furl, and support platform. The hub height of the wind turbine is $34 \mathrm{~m}$. The wind turbine has three blades with a rotor diameter of $2.9 \mathrm{~m}$ and an upwind configuration. A 48pole permanent magnet synchronous machine is simulated in Simulink to convert mechanical energy from the turbine into electric energy. One phase stator current signal is recorded for shaft rotating frequency estimation.

\section{B. Wind Speed Data}

TurbSim, which is a stochastic, full-field, turbulent wind simulator developed by the National Renewable Energy Laboratory [21], is used in the simulation study to produce wind velocity vectors in a time series across the entire rotating plane of the wind turbine's rotor. An average wind speed of $12 \mathrm{~m} / \mathrm{s}$ is chosen in the simulation study. The IEC Kaimal turbulence model is used to generate wind turbulence in all simulations. The cross-sectional area $(8 \mathrm{~m} \times 8 \mathrm{~m})$ of the wind flow is divided into a $6 \times 6$ grid where the wind velocity and direction are calculated by TurbSim for each grid cell. The output of TurbSim contains a time series of wind speed, which is used for the aeroelastic simulation in FAST. The overall structure of the simulation platform is shown in Fig. 4.

\section{Simulation Results}

Simulations are performed for the model WTG in the healthy condition (i.e., the baseline case) as well as in two imbalance fault conditions: blade imbalance and aerodynamic asymmetry. The blade imbalance is simulated by changing the mass density of one blade, which creates an uneven distribution of mass with respect to the rotor. The aerodynamic asymmetry is simulated by adjusting the pitch of one blade, which creates an uneven torque across the rotor. One phase stator current of the WTG is recorded in the simulations to estimate the shaft rotating frequency. The proposed method is then applied to extract the signatures of the faults from the recorded data in the frequency domain.

The mass density of one blade is scaled up and down in the simulations of blade imbalance. Four scenarios are simulated with the mass density of one blade adjusted by $-1 \%,+2 \%$, $-3 \%$, and $+4 \%$, while the mass densities of the other two blades are unchanged. Here, the negative sign indicates a decrease of the mass density and the positive sign indicates an increase of the mass density. The proposed method is applied to calculate the 1P-invariant PSD of the estimated shaft rotating frequency for the baseline case and the four blade imbalance scenarios. The base frequency is chosen to be $3 \mathrm{~Hz}$ (i.e., $180 \mathrm{r} / \mathrm{min}$ ) and the base value of the downsampling step size $L$ is 20 . Therefore, the variable characteristic frequency of $1 \mathrm{P}(2-4 \mathrm{~Hz})$ of the blade imbalance faults in the estimated shaft rotating frequency signal is converted to a constant value of $3 \mathrm{~Hz}$. The results are compared in Figs. 5 and 6. It is clearly shown that in the blade imbalance scenarios, excitations are evident at $1 \mathrm{P}$, which is fixed at $3 \mathrm{~Hz}$ 


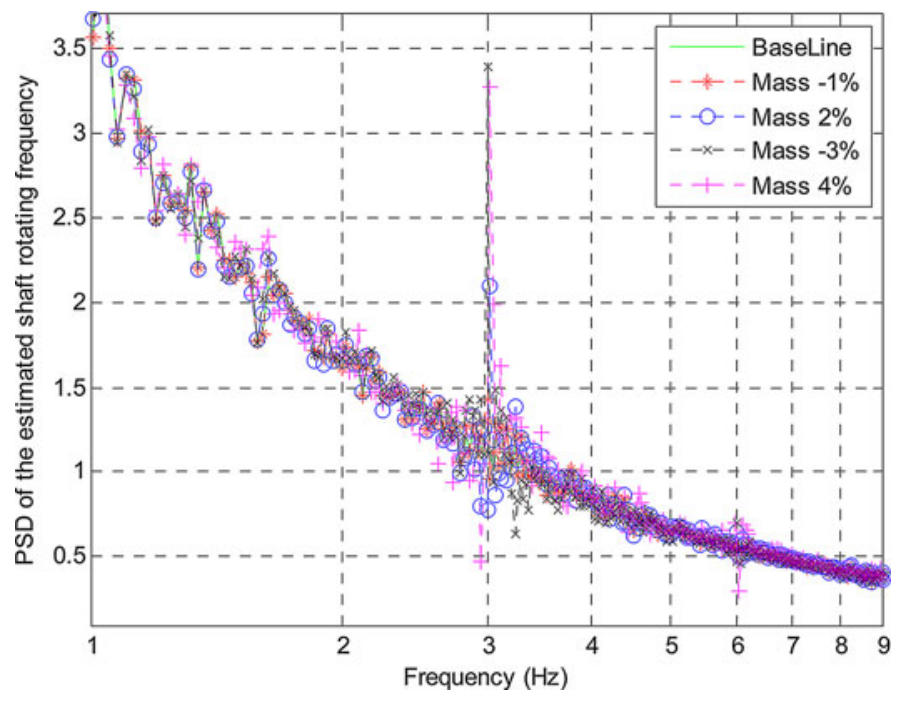

Fig. 5. Comparison of the 1P-invariant PSD of the estimated shaft rotating frequency for the blade imbalance scenarios against the baseline case in a wide frequency range.

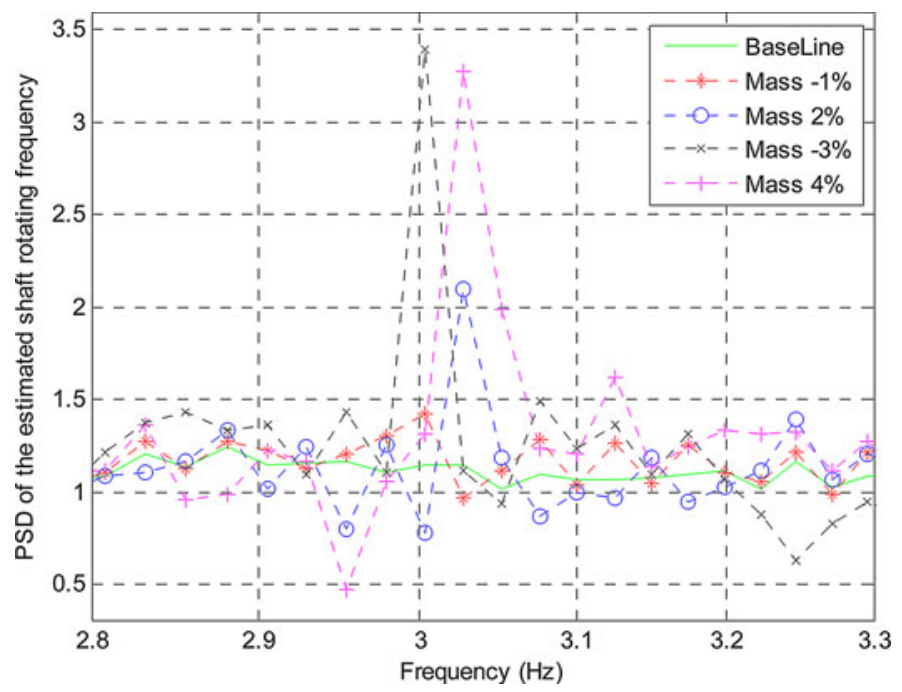

Fig. 6. Comparison of the 1P-invariant PSD of the estimated shaft rotating frequency for the blade imbalance scenarios against the baseline case in a frequency range around $1 \mathrm{P}$.

by using the proposed method. The imbalance is caused by an eccentric mass rotating with a frequency of $1 \mathrm{P}$. The shaft rotating frequency is affected by the imbalance in blades and also vibrates with a frequency of 1P. Furthermore, Fig. 6 shows that the magnitude of the excitation at $1 \mathrm{P}$ increases with the increase of the degree of blade imbalance. On the other hand, no excitation is observed at $1 \mathrm{P}$ frequency in the PSD curve for the wind turbine with healthy blades.

In order to simulate aerodynamic asymmetry faults of the wind turbine, the pitch angle of one blade is adjusted by $-2^{\circ}$, $+4^{\circ},-6^{\circ}$, and $+8^{\circ}$, while the pitch angles of the other two blades are unchanged at $11.44^{\circ}$. Figs. 7-9 compare the 1P-invariant PSD of the estimated shaft rotating frequency generated by the proposed method for the wind turbine in the four aerodynamic asymmetry scenarios against the baseline case. Again,

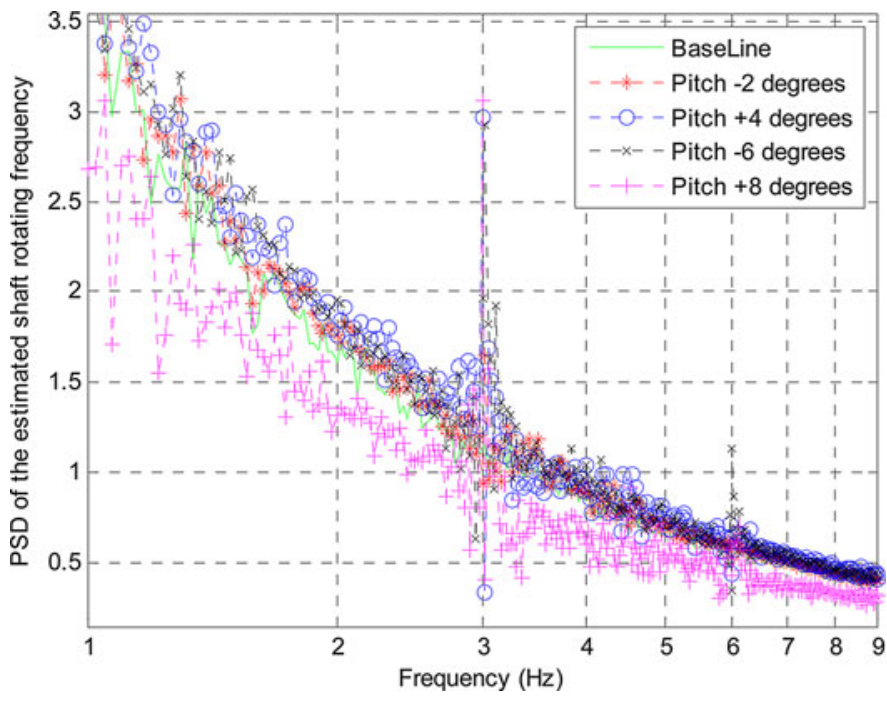

Fig. 7. Comparison of the 1P-invariant PSD of the estimated shaft rotating frequency for the aerodynamic asymmetry scenarios against the baseline case in a wide frequency range.

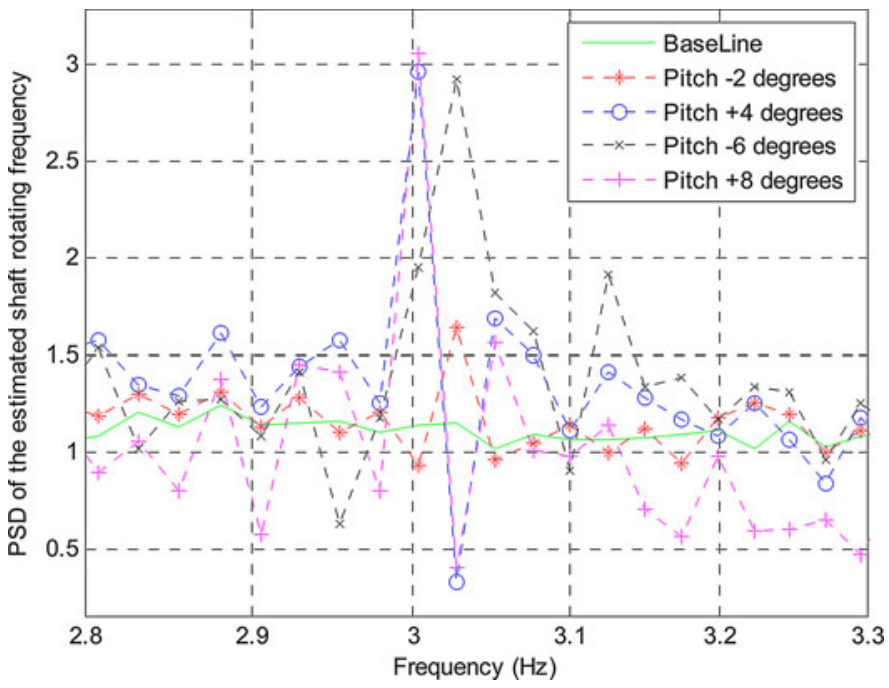

Fig. 8. Comparison of the 1P-invariant PSD of the estimated shaft rotating frequency for the aerodynamic asymmetry scenarios against the baseline case in a frequency range around $1 \mathrm{P}$.

the variable $1 \mathrm{P}$ frequency is converted to a constant value of $3 \mathrm{~Hz}$ by using the proposed method. Excitations appear at both $1 \mathrm{P}$ and $2 \mathrm{P}$ frequencies in the four aerodynamic asymmetry scenarios, which agree with the theoretical analysis in Section II. Moreover, the magnitudes of the excitations at the characteristic frequencies in the PSD plot become more significant when the degree of aerodynamic asymmetry becomes greater.

\section{EXPERIMENTAL StUdy OF IMbalance FAUlt DeteCtion}

\section{A. Experimental System Setup}

A 160-W Southwest Windpower Air Breeze direct-drive WTG is used for experimental studies. The generator has six pole pairs. The WTG is operated in a wind tunnel, as shown in Fig. 10. The wind tunnel uses a variable-speed fan to generate 


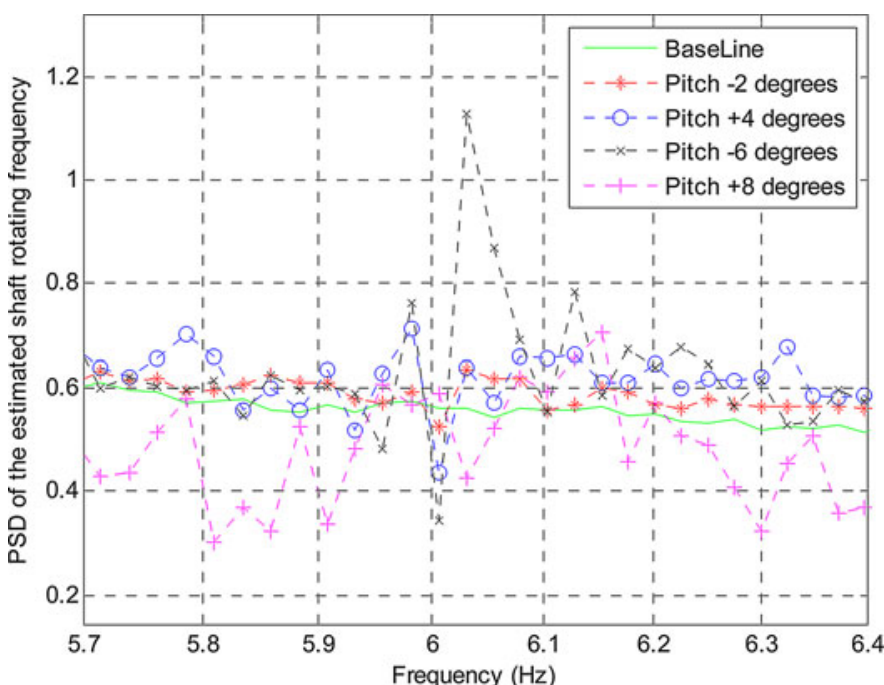

Fig. 9. Comparison of the 1P-invariant PSD of the estimated shaft rotating frequency for the aerodynamic asymmetry scenarios against the baseline case in a frequency range around $2 \mathrm{P}$

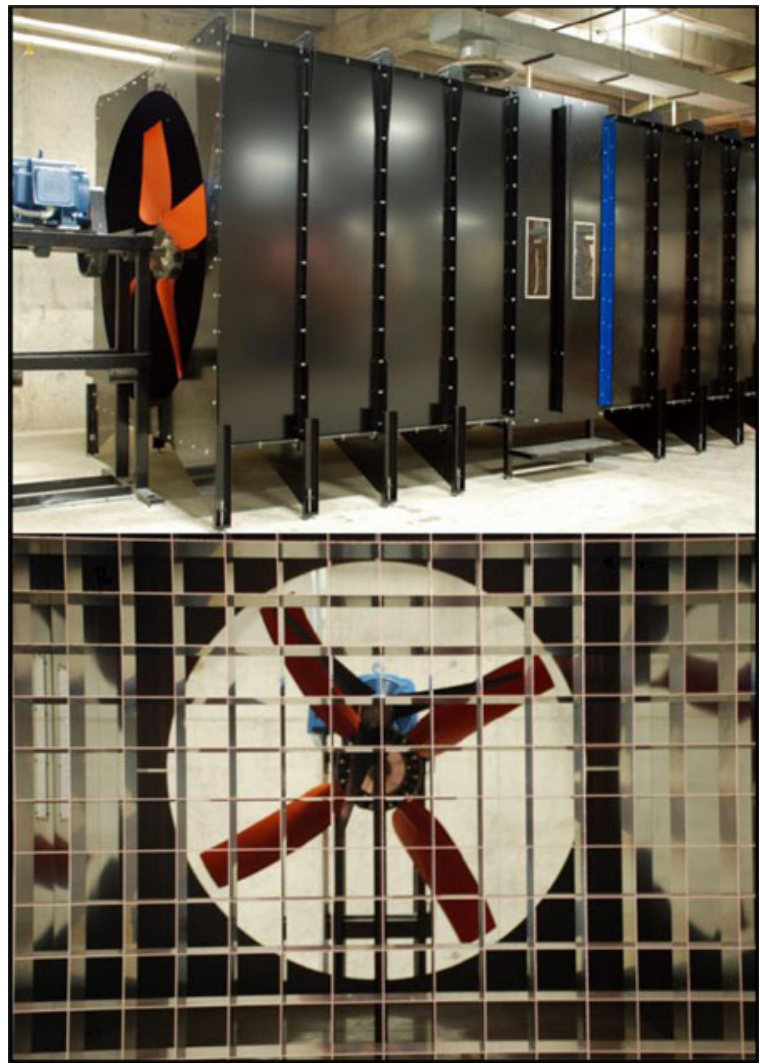

Fig. 10. Wind tunnel with the testing WTG.

controllable wind flows with the speed from 0 to $10 \mathrm{~m} / \mathrm{s}$. In the experiments, the rotating speed of the fan is varied to generate variable wind speed in the wind tunnel. One phase stator current of the WTG is recorded via a Fluke current clamp and National Instrument data acquisition system at a sampling rate of $10 \mathrm{kHz}$. The current samples are acquired by the LabView software op-

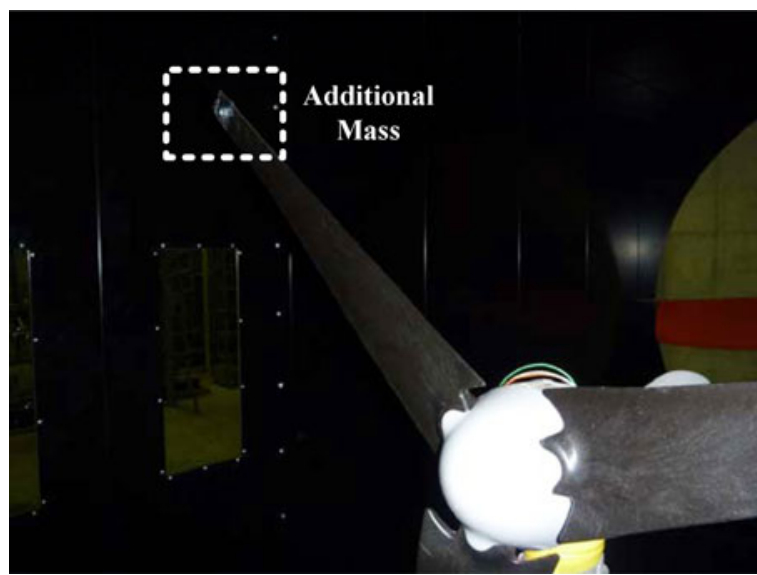

Fig. 11. Blade with an additional mass to create a blade imbalance fault.

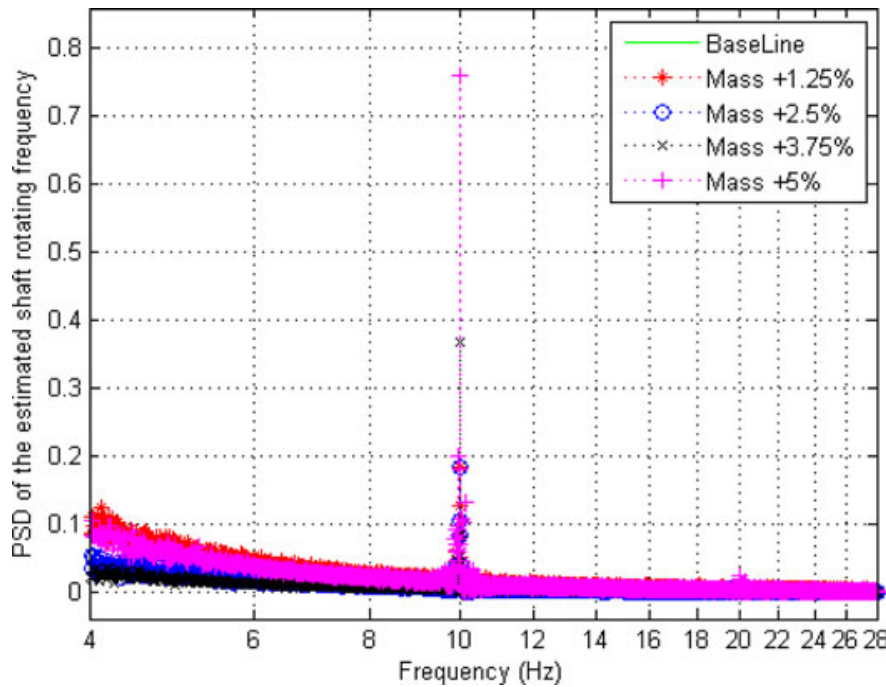

Fig. 12. Comparison of the 1P-invariant PSD of the estimated shaft rotating frequency for the blade imbalance scenarios against the baseline case in a wide frequency range.

erating on a lab computer. The length of each current record is $60 \mathrm{s.}$

\section{B. Detection of Blade Imbalance}

To create a blade imbalance, additional masses are added close to the tip of a blade of the WTG, as shown in Fig. 11. The mass of a healthy blade is measured to be $181 \mathrm{~g}$. Four blade imbalance scenarios are tested by adding a mass of 2.3, 4.5, 6.8, and $9 \mathrm{~g}$, respectively, to a blade. Therefore, the weight of the blade is increased by $1.25 \%, 2.5 \%, 3.75 \%$, and $5 \%$, respectively. During the experiments, the WTG is operated at variable speed in the range of $6-13 \mathrm{~Hz}$, which is the variable $1 \mathrm{P}$ frequency.

The proposed method is applied to obtain the 1P-invariant PSD of the estimated shaft rotating frequency of the WTG for the four blade imbalance scenarios and the baseline case. In the proposed method, the base frequency $f_{b}$ is chosen to be $10 \mathrm{~Hz}$ and the base value of the downsampling step size $L$ is 100 . The results are compared in Figs. 12 and 13, where excitations are clearly observed at the fixed $1 \mathrm{P}$ frequency of $10 \mathrm{~Hz}$ in the 


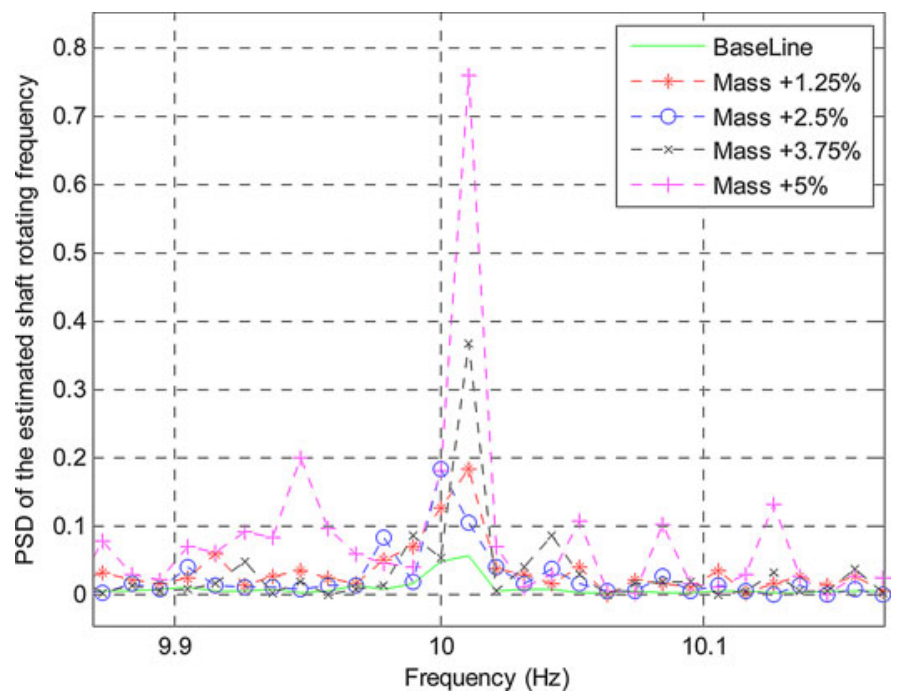

Fig. 13. Comparison of the 1P-invariant PSD of the estimated shaft rotating frequency for the blade imbalance scenarios against the baseline case in a frequency range around $1 \mathrm{P}$.

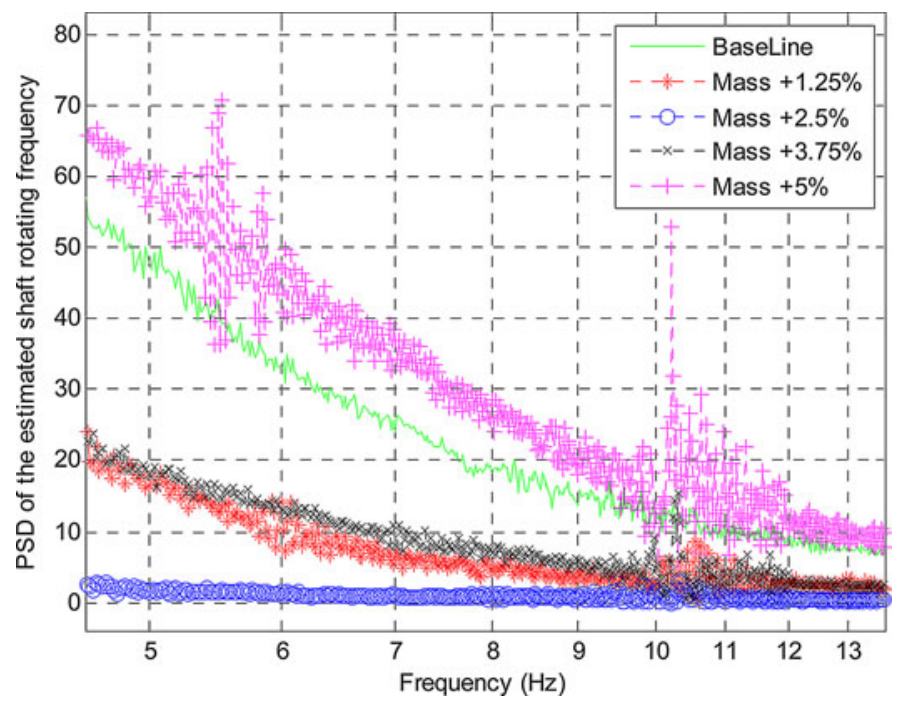

Fig. 14. Comparison of the PSD of the estimated shaft rotating frequency obtained directly from the standard PSD analysis for the blade imbalance scenarios against the baseline case.

blade imbalance scenarios. Thus, the magnitude of this excitation provides an effective index for detecting blade imbalance faults. The greater the magnitude of the excitation appears at the 1P frequency, the higher degree of the blade imbalance. Therefore, the proposed method can not only identify but can also quantify the degree of blade imbalance of the WTG.

The PSD of the estimated shaft rotating frequency is also calculated by using the standard PSD analysis directly without using the upsampling and downsampling algorithms in the proposed method. The results are compared in Fig. 14 for the blade imbalance scenarios against the baseline case. The PSD of the estimated shaft rotating frequency excites at $1 \mathrm{P}$ frequency in the range of $6-13 \mathrm{~Hz}$ in the blade imbalance scenarios. However, it is difficult to quantify and evaluate the blade imbalance faults by using these results due to the variations of the $1 \mathrm{P}$ frequency

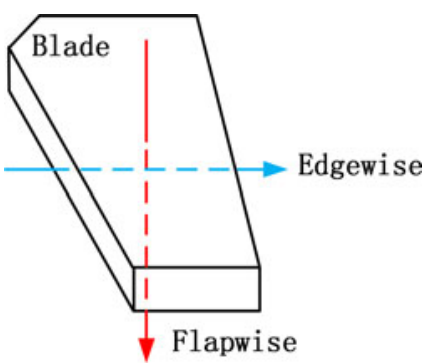

Fig. 15. Blade bended flapwise or edgewise.

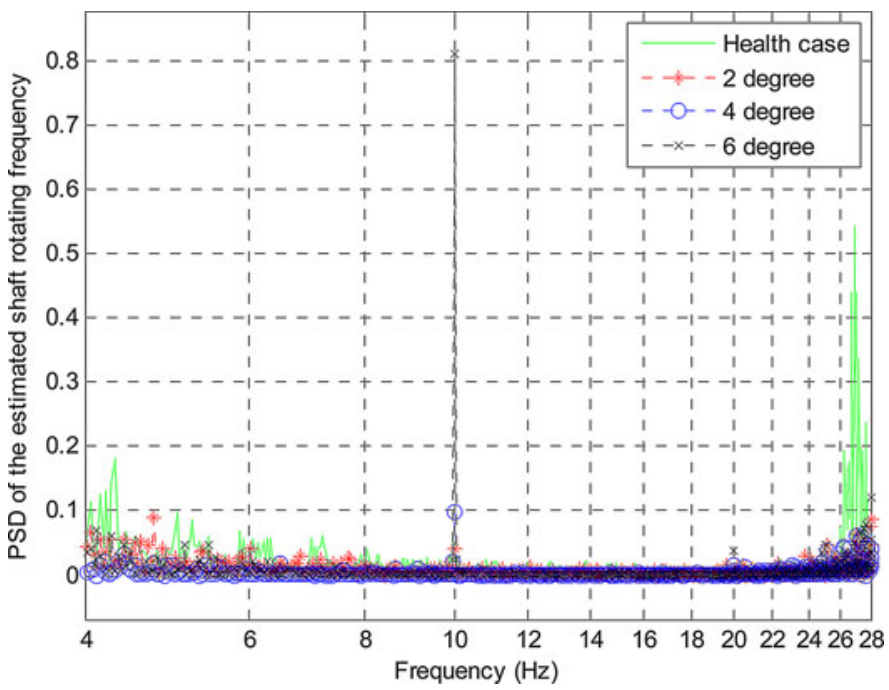

Fig. 16. Comparison of the 1P-invariant PSD of the estimated shaft rotating frequency for the bent blade scenarios against the baseline case in a wide frequency range.

component. Furthermore, if interferences are present near the $1 \mathrm{P}$ frequency, it will be difficult to identify fault signatures from the interferences by using the standard PSD analysis.

\section{Detection of Bent Blade}

A bent blade is a blade of a WTG that twists flapwise or edgewise, as illustrated in Fig. 15. A bent blade also generates an imbalance fault in the WTG. During the experiments, one blade of the Air Breeze wind turbine is bent edgewise at $2^{\circ}, 4^{\circ}$, and $6^{\circ}$, respectively.

Figs. 16 and 17 compare the 1P-invariant PSD of the estimated shaft rotating frequency of the WTG for the bent blade scenarios against the baseline case. The 1P-invariant PSD is obtained by using the proposed method with the same base frequency $f_{b}$ and base value of the downsampling step size $L$ as in the blade imbalance study. As shown in Figs. 16 and 17, an excitation appears at a fixed frequency of $1 \mathrm{P}(10 \mathrm{~Hz})$ in the PSD plots of the bent blade cases. The magnitude of the 1P excitation provides an effective index for detecting and quantifying the bent blade faults.

Since the wind turbine is operated in the wind tunnel during the experiments and there is no wind shear or yaw error in the wind tunnel, as shown in Figs. 12 and 16, there is no excitation 


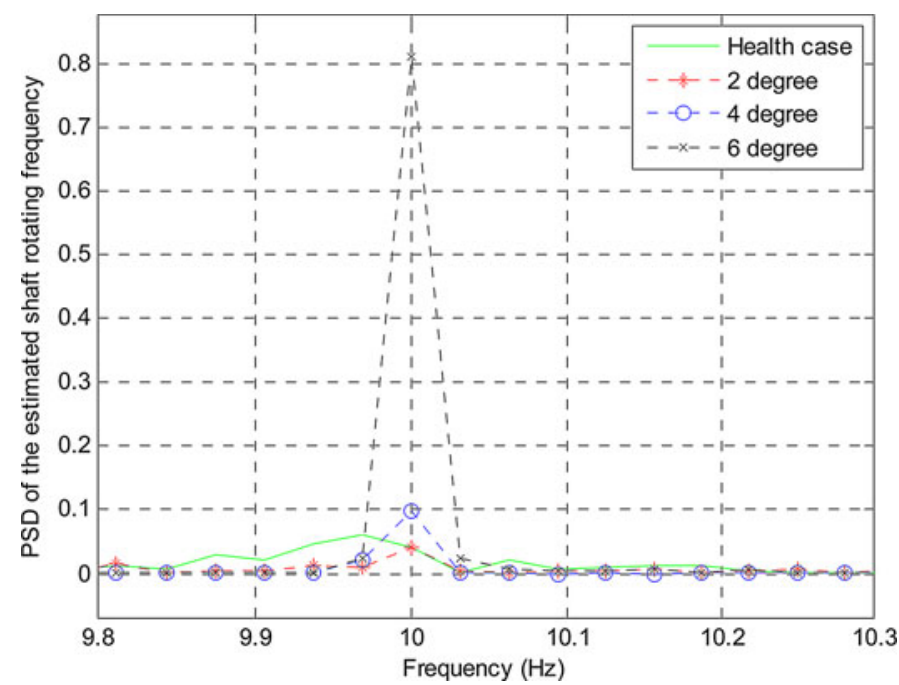

Fig. 17. Comparison of the 1P-invariant PSD of the estimated shaft rotating frequency for the bent blade scenarios against the baseline case in a frequency range around $1 \mathrm{P}$.

at $2 \mathrm{P}$ frequency in the experimental results, which is another characteristic frequency of aerodynamic asymmetries.

\section{CONCLUSION}

This paper has proposed a novel method for imbalance fault detection and quantification of direct-drive wind turbines using only one phase generator stator current signal. The proposed method firstly estimates the shaft rotating frequency of the WTG from the generator current record by using a PLL algorithm, and then processes the estimated shaft rotating frequency signal in a way such that the variable characteristic frequencies of the imbalance faults become constant values in the PSD of the processed shaft rotating frequency signal. Simulation studies have been carried out in a FAST and Simulink combined environment for blade imbalance and aerodynamic asymmetry detection of a 10-kW direct-drive WTG. Experimental studies have been performed in a wind tunnel facility for blade imbalance and bent blade detection of a $160-\mathrm{W}$ direct-drive WTG. Both simulation and experimental results have confirmed that the proposed method is effective to detect and quantify various imbalance faults in a variable-speed direct-drive WTG by only using one phase current measured from the generator terminal.

The proposed method is nonintrusive, because it only uses one phase stator current signal, which has been used in existing WTG control systems; no additional sensors or data acquisition devices are needed. The proposed method is able to clearly identify excitations at the characteristic frequencies of imbalance faults. Therefore, it is sensitive to faults and is immune from interferences near fault characteristic frequencies. Compared to other signal analysis methods, such as wavelet analysis, amplitude demodulation, etc., the proposed method is less complex and has a lower computational cost and, therefore, is good for online fault detection. Furthermore, the traditional PSD analysis is a well-developed method for fault detection of rotating machines. Therefore, the proposed 1P-invariant PSD method can be easily integrated into existing condition monitoring and fault detection systems used in the wind industry. Future work will focus on identifying the types of imbalance faults and prognosing WTG health condition by using the fault features extracted.

\section{REFERENCES}

[1] The American Wind Energy Association. (2011, Apr.) U.S. Wind Industry First Quarter 2011 Market Report. [Online]. Available: http://www.awea.org/learnabout/publications/reports

[2] Y. Amirat, M. E. H. Benbouzid, B. Bensaker, and R. Wamkeue, "Condition monitoring and fault diagnosis in wind energy conversion systems: A review," in Proc. IEEE Int. Electr. Mach. Drives Conf., May 2007, vol. 2, pp. 1434-1439.

[3] S. A. Saleh and C. R. Moloney, "Development and testing of wavelet packet transform-based detector for ice accretion on wind turbines," in Proc. IEEE Digital Signal Process. Workshop IEEE Signal Process. Education Workshop, Jan. 2011, pp. 72-77.

[4] R. W. Hyers, J. G. McGowan, K. L. Sullivan, J. F. Manwell, and B. C. Syrett, "Condition monitoring and prognosis of utility scale wind turbines," Energy Mater., vol. 1, no. 3, pp. 187-203, Sep. 2006.

[5] B. Lu, Y. Li, X. Wu, and Z. Yang, "A review of recent advances in wind turbine condition monitoring and fault diagnosis," presented at the IEEE Symp. Power Electron. Mach. Wind Appl., Lincoln, NE, Jun. 2009.

[6] R. Ramlau and J. Niebsch, "Imbalance estimation without test masses for wind turbines," J. Solar Energy Eng., vol. 131, no. 1, pp. 011010-1011010-7, Feb. 2009.

[7] D. Jiang, Q. Huang, and L. Hong, "Theoretical and experimental study on wind wheel unbalance for a wind turbine," in Proc. World Non-GridConnected Wind Power Energy Conf., Sep. 2009, pp. 1-5.

[8] M. Zhao, D. Jiang, and S. Li, "Research on fault mechanism of icing of wind turbine blades," in Proc. World Non-Grid-Connected Wind Power Energy Conf., Sep. 2009, pp. 1-4.

[9] C. S. Tsai, C. T. Hsieh, and S. J. Huang, "Enhancement of damage detection of wind turbine blades via CWT-based approaches," IEEE Trans. Energy Convers., vol. 21, no. 3, pp. 776-781, Sep. 2006.

[10] W. Q. Jeffries, J. A. Chambers, and D. G. Infield, "Experience with bicoherence of electrical power for condition monitoring of wind turbine blades," IEE Proc.: Vision, Image Signal Process., vol. 145, no. 3, pp. 141148, Jun. 1998.

[11] R. Fadaeinedjad, G. Moschopoulos, and M. Moallem, "The impact of tower shadow, yaw error, and wind shears on power quality in a wind diesel system," IEEE Trans. Energy Convers., vol. 24, no. 1, pp. 102 111, Mar. 2009.

[12] E. Al Ahmar, V. Choqueuse, M. E. H. Benbouzid, Y. Amirat, J. El Assad, R. Karam, and S. Farah, "Advanced signal processing techniques for fault detection and diagnosis of a wind turbine induction generator drive train: A comparative study," in Proc. IEEE Energy Convers. Congr. Expo., Sep. 2010, pp. 3576-3581.

[13] Y. Amirat, V. Choqueuse, and M. E. H. Benbouzid, "Condition monitoring of wind turbines based on amplitude demodulation," in Proc. IEEE Energy Convers. Congr. Expo., Sep. 2010, pp. 2417-2421.

[14] V. Choqueuse, M. E. H. Benbouzid, Y. Amirat, and S. Turri, "Diagnosis of three-phase electrical machines using multidimensional demodulation techniques," IEEE Trans. Ind. Electron., vol. 59, no. 4, pp. 2014-2023, Apr. 2012.

[15] A. Kusiak and A. Verma, "A data-driven approach for monitoring blade pitch faults in wind turbines," IEEE Trans. Sustainable Energy, vol. 2, no. 1, pp. 87-96, Jan. 2011.

[16] T. Burton, D. Sharpe, N. Jenkins, and E. Bossanyi, Wind Energy Handbook. New York: Wiley, 2001.

[17] D. J. Gardels, W. Qiao, and X. Gong, "Simulation studies on imbalance faults of wind turbines," in Proc. IEEE Power Energy Soc. General Meeting, Jul. 2010, pp. 1-5.

[18] J. F. Manwell, J. G. McGowan, and A. L. Rogers, Wind Energy Explained West Sussex, U.K: Wiley, 2002.

[19] W. Qiao, W. Zhou, J. M. Aller, and R. G. Harley, "Wind speed estimation based sensorless output maximization control for a wind turbine driving a DFIG," IEEE Trans. Power Electron., vol. 23, no. 3, pp. 1156-1169, May 2008.

[20] J. M. Jonkman and M. L. Buhl, FAST User's Guide. Golden, CO: Natl. Renewable Energy Lab., Jul. 2005.

[21] B. J. Jonkman, TurbSim User's Guide. Golden, CO: Nat. Renewable Energy Lab., 2009. 


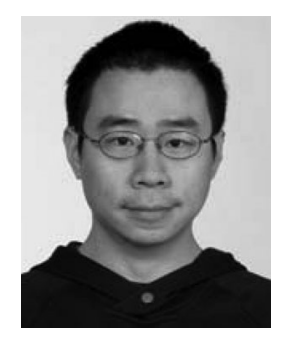

Xiang Gong (S'09) received the B.Eng. degree in electrical engineering from Huazhong University of Science and Technology, Wuhan, China, in 2006, and the M.Eng. degree in electrical engineering from Zhejiang University, Hangzhou, China, in 2008. He is currently working toward the Ph.D. degree in the Department of Electrical Engineering, University of Nebraska-Lincoln, Lincoln.

His research interests include renewable energy systems, power electronics, and condition monitoring and fault detection of wind turbine generators.

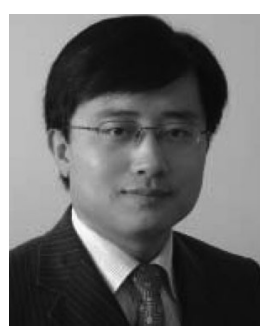

Wei Qiao (S'05-M'08) received the B.Eng. and M.Eng. degrees in electrical engineering from Zhejiang University, Hangzhou, China, in 1997 and 2002, respectively, the M.S. degree in high performance computation for engineered systems from Singapore-MIT Alliance, Singapore, in 2003, and the Ph.D. degree in electrical engineering from the Georgia Institute of Technology, Atlanta, in 2008.

From 1997 to 1999, he was an Electrical Engineer with China Petroleum \& Chemical Corporation (Sinopec). He is currently the Harold and Esther Edgerton Assistant Professor with the Department of Electrical Engineering, University of Nebraska-Lincoln (UNL), Lincoln. He is the author or coauthor of three book chapters and more than 90 papers in refereed journals and international conference proceedings. His research interests include renewable energy systems, smart grids, microgrids, power system control and optimization, condition monitoring and fault diagnosis, energy storage systems, power electronics, electric machines and drives, and computational intelligence for electric power and energy systems.

Dr. Qiao is an Associated Editor of the IEEE TRANSACTIONS ON INDUSTRY APPLICATIONS, the Chair of the Technical Thrust of Sustainable Energy Sources of the IEEE Power Electronics Society, and the Chair of the Task Force on Intelligent Control for Wind Plants of the IEEE Power and Energy Society. He is the Publications Chair of the 2013 IEEE Energy Conversion Congress and Exposition and the Technical Program Co-Chair and Publications Chair of the 2012 IEEE Symposium on Power Electronics and Machines in Wind Applications (PEMWA 2012). He was the Technical Program Co-Chair and Finance Co-Chair of PEMWA 2009. He was the recipient of a 2010 National Science Foundation CAREER Award, the 2010 IEEE Industry Applications Society Andrew W. Smith Outstanding Young Member Award, the 2011 UNL Harold and Esther Edgerton Junior Faculty Award, and the 2011 UNL College of Engineering Edgerton Innovation Award. 